\title{
Norois
}

Environnement, aménagement, société

$198 \mid 2006 / 1$

Géosymbole, écologie, renouvellement urbain, modélisation

\section{Modélisation prospective de l'occupation du sol en zone agricole intensive dans la France de l'Ouest}

Prospective modeling of land use in intensive agriculture area in western part of

France

\section{Thomas Houet}

\section{OpenEdition}

Journals

\section{Édition électronique}

URL : https://journals.openedition.org/norois/2025

DOI : $10.4000 /$ norois. 2025

ISBN : 978-2-7535-1546-8

ISSN : 1760-8546

Éditeur

Presses universitaires de Rennes

Édition imprimée

Date de publication : 1 mars 2006

Pagination : $35-47$

ISBN : 978-2-7535-0307-6

ISSN : 0029-182X

Référence électronique

Thomas Houet, « Modélisation prospective de l'occupation du sol en zone agricole intensive dans la France de l'Ouest », Norois [En ligne], 198|2006/1, mis en ligne le 13 décembre 2008, consulté le 14 janvier 2022. URL : http://journals.openedition.org/norois/2025; DOI : https://doi.org/10.4000/norois. 2025 


\title{
MODÉLISATION PROSPECTIVE DE L'OCCUPATION DU SOL EN ZONE AGRICOLE INTENSIVE DANS LA FRANCE DE L'OUEST
}

\author{
Thomas Houet \\ COSTEL - Université Rennes 2 Haute-Bretagne \\ LETG, UMR 6554 - CNRS \\ thomas.houet@uhb.fr
}

\section{RÉSUMÉ}

La réforme de la Politique Agricole Commune programmée en 2006 et la dévitalisation des campagnes provoquant un agrandissement prononcé des exploitations agricoles soulèvent la problématique de l'évolution des modes d'occupation des sols et de ses conséquences sur l'environnement. L'élaboration de scénarios prospectifs spatialisés à l'échelle locale constitue une méthode puissante pour donner une image des futurs possibles d'un territoire agricole. Lorsque celui-ci est porteur d'enjeux sur la gestion qualitative de l'eau (tel qu'un bassin versant), ces images peuvent aider les décideurs à mettre en place des actions de gestion de la ressource efficaces localement et durables. L'objectif de ce travail est d'évaluer l'influence respective de la réforme de la PAC et de l'agrandissement des exploitations agricoles sur les changements d'occupation des sols à l'aide de simulations dynamiques effectuées avec une plateforme de modélisation. Les résultats montrent que ces deux facteurs ont des conséquences différenciées sur les changements d'occupation des sols à l'échelle d'un petit bassin versant (1 $300 \mathrm{ha}$ ) : la direction, le rythme et la distribution spatiale des changements d'occupation et d'utilisation des sols diffèrent.

MotS CLÉS: Changements d'occupation du sol-Exploitation agricole - Prospective spatialisée - Scénario - Simulations dynamiques - successions culturales.

\section{ABSTRACT \\ Prospective modeling of land use in intensive agriculture area in western part of France}

The reform of the CAP which should come into force in 2006 and the depopulation in rural countries that induces significant enlargement of farming units refer to the problematic of land use, land cover changes and their environmental impacts. Construction of prospective scenarios spatialized at a fine scale is a powerful method to provide alternative future images of an agricultural area. When these areas are concerned by water quality management (such as a watershed), these images may help decision-makers to implement efficient and sustainable water management policies. The aim of this paper is to evaluate respective influence of the 2006's CAP reform and of farming units extensions on land use changes from dynamic simulations based on a modeling platform. Results show that these two factors have differentiated consequences on land use and cover changes for watershed of small extend ( $1300 \mathrm{ha}$ ): direction, rythm and spatial distribution of land-use and landcover changes differ. 
KEY WORDS : Crop Successions - Dynamic Simulations - Farm - Landcover Changes

- Scenario - Spatial Prospective.

Les changements actuels des usages des sols en région agricole intensive reflètent des stratégies d'acteurs multiples qui se situent à des échelles spatiales très différentes (Europe, Etat, Région, Commune, bassin versant). L'ensemble des variables économiques (Politique Agricole Commune, GATT $^{1}, \mathrm{OMC}^{2}$, marchés, proximité des industries agro-alimentaires, etc.) et environnementales (Mesures Agri-Environnementales, Directive Cadre sur l'Eau, Schéma d'Aménagement et de Gestion des Eaux, Contrat Territoriaux d'Exploitation relayés par les Contrats d'Agriculture Durable, etc.) agissent de façon directe ou indirecte à l'échelle de l'exploitation agricole (Corgne, 2005 ; Houet et Hubert-Moy, 2004). L'agriculteur intègre l'ensemble de ces variables par les choix d'assolements qu'il réalise d'une année sur l'autre ou dans une vision à plus long terme afin de répondre à ses objectifs économiques, voire environnementaux.

Les types d'occupation du sol au sein d'une exploitation agricole dépendent essentiellement du système de production adopté par l'exploitant. Suivant une typologie qui peut être plus ou moins détaillée, chaque système de production se caractérise par un assolement lui-même défini par les proportions de chacune des cultures principales réparties sur la surface agricole utile. Dans les régions d'élevage telle que la Bretagne, les prairies temporaires (ray-grass anglais ou italien), le maïs, les céréales et oléo-protéagineux constituent les cultures principales les plus courantes. Les exploitants agricoles raisonnent l'utilisation de leurs parcelles en fonction des conditions physiques du milieu (pente, type de sol, degré d'hydromorphie, etc.), mais aussi en fonction de l'organisation de leur travail (distance au siège d'exploitation, taille des parcelles, éclatement du parcellaire de l'exploitation, successions agronomiques) (Corgne, 2005). Les travaux de C. Thenail (1997) illustrent les modifications du système technique de production et de la gestion territoriale de l'exploitation dès lors qu'un évènement intervient (réforme, changement d'exploitant, etc.). Parmi ces évènements, les réformes de la PAC et les changements des structures foncières des exploitations constituent des moteurs forts dans les changements d'usage des terres. Toutefois, l'impact de ces évènements sur l'évolution de l'occupation du sol est assez mal connu, bien qu'ils participent pour une part non négligeable à la dégradation ou à l'amélioration des milieux, notamment à la qualité des sols et de la ressource en eau.

L'élaboration de scénarios prospectifs constitue une méthode puissante pour s’interroger sur les devenirs possibles d'un territoire, et aider à la prise de décisions (Poux, 2003; Mermet et Poux, 2002; Poux et al., 2001). L'objectif de ce travail est d'évaluer quantitativement et spatialement l'influence respective de la réforme de la PAC et de l'agrandissement des exploitations agricoles sur les changements d'occupation des sols à l'échelle d'un bassin versant à l'aide de simulations dynamiques effectuées avec une plateforme de modélisation. Les simulations sont réalisées sur un bassin versant localisé en Bretagne centrale, le Lestolet, faisant l'objet d'un programme de reconquête de la qualité de l'eau, dans un contexte agricole où l'élevage bovin est dominant (fig. 1 - planche I). La localisation de zones à risque vis-à-vis des transferts de flux constitue un enjeu fort pour la préservation à moyen et long terme de la ressource en eau.

Après avoir succinctement exposé comment la PAC 2006 et les modifications des structures foncières pourraient orienter l'évolution de l'occupation des sols à l'échelle d'une exploitation, l'expérience de modélisation permettant de produire des simulations selon plusieurs scénarios contrastés sur le site d'étude est présentée et les premiers résultats relatifs à l'impact respectif de la PAC et du foncier sur l'occupation des sols sont montrés.

2. Organisation Mondiale du Commerce. 


\section{La PAC et l'évolution de la structure foncière des exploitations agricoles, facteurs d'évolution de l'occupation des sols}

Il s'agit de présenter l'impact de l'évolution des exploitations sur les types d'occupation des sols à travers l'évolution des assolements liée à la réforme de la PAC prévue en 2006 et leur agrandissement foncier.

\section{LA RÉFORME DE LA PAC DE 2006 ET SON INFLUENCE SUR L'OCCUPATION DES SOLS}

\section{À L'ÉCHELLE DE L'EXPLOITATION AGRICOLE}

La réforme de la PAC constitue un facteur agissant sensiblement sur l'évolution des modes d'occupation des sols. Elle engendre des contraintes économiques auxquelles l'exploitant s'adapte, par exemple en modifiant son assolement. Les primes attribuées aux céréales et aux maïs fourragers dans le cadre de la PAC de 1992 se sont ainsi traduites en Bretagne par une augmentation de la part des cultures au détriment des prairies temporaires et naturelles mais suivant des ampleurs variables pour des secteurs aux paysages agraires et aux productions dominantes différentes (Hubert-Moy et al., 2003).

La prochaine réforme de la PAC annoncée pour 2006 soulève aujourd'hui la problématique de son influence sur l'évolution des types d'occupation des sols d'un territoire (bassin de production, bassin versant, etc.) et de son incidence environnementale. Les stratégies d'adaptation des exploitations du Grand Ouest à la PAC 2006 et leurs impacts sur les types d'occupation des sols, le bocage et les zones humides, ont été déduites lors d'une réunion regroupant des experts ${ }^{3}$ en agronomie, en prospective, en écologie du paysage, sur l'agriculture bretonne, sur les zones humides et des gestionnaires de l'eau et de l'espace agricole. Ces stratégies ont ensuite été confortées à l'aide d'enquêtes de terrains ayant concernées 167 exploitations dispersées sur les quatre départements bretons (Geslot, 2005) et par des experts de l'ONIC ${ }^{4}$.

Pour une exploitation, deux stratégies antagonistes sont envisageables :

- Dans le premier cas, la PAC incite à l'extension des systèmes herbagers, ce qui est synonyme d'une future désintensification de la production agricole. Le découplage total des aides permettrait alors aux exploitants de percevoir la même somme à l'hectare (à + ou $-25 \%$ ) quels que soient les couverts implantés. Par exemple, dans le cas d'élevages laitiers et/ou allaitant, l'exploitant ayant beaucoup de terres pourrait faire le choix de baisser ses coûts de production par l'implantation d'herbages (passage à une gestion plus extensive). Dans le cas où l'exploitant est relativement proche de la retraite, n'a ni emprunt à rembourser ni repreneur, celui-ci pourrait tirer un revenu convenable de son activité et réduire considérablement sa charge de travail en implantant des prairies pour lesquelles les primes n'exigent qu'un entretien minimal (fauche annuelle ou semestrielle).

- Dans le second cas, la PAC favorise l'implantation de céréales, ce qui se traduit par une intensification de la production. Dans le cas d'élevages laitiers, les exploitants ayant peu de terres, pourraient faire baisser le coût de leur production en augmentant la production laitière par vache. Ils devraient alors réduire légèrement leur cheptel augmentant ainsi la part de maïs fourrager par vache. Les surfaces en blé et autres céréales pouvant quant à elles légèrement augmenter afin d'assurer le versement de $100 \%$ des aides « grandes cultures ». Dans le cas d'exploitations n'ayant plus d'emprunts à rembourser et devant mettre leurs bâtiments d'élevages aux normes, les élevages pourraient disparaître, des céréales pouvant être implantées sur l'ensemble des terres labourables. Leur revenu proviendrait alors des marges réalisées sur les céréales à fortes valeurs ajoutées (blé, colza, chanvre, lin, etc.) ou d'autres cultures (légumes industriels, etc.).

3. Les experts sollicités sont Xavier Poux (AScA/RGTE CIRED-ENGREF), Jacques Baudry (INRA-SAD Armorique) et Bernard Clément (ECOBIO).

4. Office National Interprofessionnel des Céréales - Experts sollicités : Yves Bonhomme et Stéphanie Geslot. 


\section{L'IMPACT DES CHANGEMENTS DE LA STRUCTURE FONCIÈRE DES EXPLOITATIONS}

Peu de recherches portent sur l'impact des changements de la structure foncière des exploitations sur l'usage des terres car les mécanismes qui régissent ces changements sont très mal connus. De plus, les données sur la structure foncière actuelle des exploitations sont difficilement accessibles. En outre, ces données ont été élaborées de façon très ponctuelle avant la mise en place récente des déclarations PAC à partir d'orthophotoplans ou des diagnostics phytosanitaires du territoire des exploitations, ce qui ne permet pas d'étudier précisément et de façon exhaustive les dynamiques du foncier agricole.

La Bretagne connaît une forte diminution de sa population active agricole en Bretagne. Le nombre d'exploitations était de 92000 en 1988, contre 51200 en 2000 (Agreste Bretagne, 1998; 2001). Leur taille moyenne est passée de 33 ha en 2000 contre 19 ha en 1988. Dans un temps comparable (1985-1997), la proportion de surfaces en herbe (prairies temporaires et permanentes) par rapport à la SAU bretonne est passée de 47,3\% en 1985 à 39,5\% en 1997; celle des cultures (céréales + maïs fourrager) de 39,1\% à 48 \% (Agreste Bretagne, 1998). Ainsi, si l'agrandissement des exploitations a accompagné la hausse des cultures, l'existence d'une relation de causalité n'a jamais été démontrée. D’autres facteurs, comme la réforme de la PAC de 1992 où encore les quotas laitiers, sont mis en avant pour expliquer cette évolution (Houet et Hubert, 2004).

Parmi les quelques travaux portant sur l'impact des changements des structures foncières sur l'utilisation des terres, ceux de C. Thenail et J. Baudry (2001) et J. Baudry et al., (2003) ont montré que l'exploitant est amené à adapter son assolement et ses règles d'allocation des cultures en fonction de la configuration spatiale de son exploitation (importance de sa surface agricole utile, distribution spatiale, taille et géométrie de ses parcelles en plus de leurs caractéristiques agronomiques). En résumé, les changements de la structure foncière des exploitations modifient les règles d'usages des terres (allocation spatiale des cultures, utilisation des parcelles humides, etc.). Mais les conséquences sur l'occupation des sols à l'échelle d'un territoire agricole (par exemple un bassin versant) n’ont pas encore été mesurées.

\section{Méthodologie}

Les simulations dynamiques et spatialement explicites sont effectuées sur le site du Lestolet à l'aide d'une plateforme de modélisation de paysages (L1) suivant des scénarios prospectifs contrastés. En fonction des stratégies d'adaptation à la réforme de la PAC 2006 et des modifications des structures foncières envisageables sur le site d'étude, trois scénarios ont été construits afin d'éclairer leur influence probable sur l'occupation agricole des sols à l'échelle du bassin versant. Le premier prolonge les évolutions actuellement observées; le second est construit sur une hypothèse d'évolution vers un système herbager sous l'impulsion de la PAC du fait de la situation actuelle des exploitations du Lestolet sans prendre en compte les changements fonciers; enfin le dernier fait évoluer les structures foncières sans considérer les changements d'assolement liés à la réforme de la PAC.

\section{Présentation du site d’étude}

Le bassin versant du Lestolet, d'une superficie totale de 1320 ha, est situé dans le Sud-Ouest du département des Côtes-d'Armor, en Centre Bretagne (fig. 1-planche I). Territoire au bocage dense (124 m/ha en 1998) et aux zones humides de fonds de vallées très présentes (17 \% du bassin versant), l'utilisation du sol est quasi-exclusivement agricole. Les communes couvrant le bassin versant s'inscrivent dans une dynamique de dévitalisation prononcée de son tissu démographique : leur densité de population baisse de -10 à -40\% entre 1982 et 1999, avec moins de $30 \mathrm{hab} / \mathrm{km}^{2}$ en 1999 (INSEE, 1982 ; INSEE, 1999), et leur population agricole vieillit avec moins de $20 \%$ d'actifs agricoles de plus de 55 ans en 1979 contre près de 60 \% en 1999 (ONIC, 1979; ONIC, 2000). 
En 1998, 31 exploitations sont connues pour avoir tout ou partie de leurs terres sur le Lestolet ${ }^{5}$. Ce site se spécialise depuis le début des années 1980 dans l'élevage bovin, la production avicole ayant été affectée par les crises des années 1990 (Houet et Hubert-Moy, 2004). Les exploitations «bovin lait/viande » sont largement dominantes (20) par rapport aux exploitations mixtes « bovin + volailles » (7); l'élevage hors-sol porcin ou avicole est peu présent (1 exploitation de chaque); les exploitations mixtes « bovin + porcs » sont peu nombreuses (2). Cela se traduit par une dominance des prairies temporaires (473 ha) et permanentes (178 ha) et des superficies en maïs et céréales plus faibles (respectivement 224 et 198 ha).

Enfin, le Lestolet fait l'objet d'un programme de reconquête de la qualité de l'eau (Bretagne Eau Pure) suite à la dégradation de ses eaux par les nitrates, qui a contribué à la mise aux normes des bâtiments d'élevage dans le cadre des Plans de Maîtrise des Pollutions d'Origine Agricole (PMPOA 1 et 2).

\section{MODÉLISATION À TRAVERS LA PLATEFORME L1}

\section{La plateforme L1}

La plateforme de modélisation L1 se présente comme un noyau commun de données, de connaissances et de méthodes (empiriques, statistiques, etc.) autour duquel gravitent des modèles (sous la forme de modules) dédiés à différentes applications (fig. 2 - planche II). La comparaison des plateformes de modélisation existantes en matière de simulation dynamique du paysage (Gaucherel et al., soumis; Houet et Gaucherel, sous presse) a montré que la spécificité de L1 tient dans la description discontinue et évolutive du paysage agraire sur le plan géométrique et attributaire, dans l'intégration des interactions entre des variables représentées à différentes échelles, d'une gestion souple de la dynamique temporelle (tous les pas de temps possibles) et d'une modélisation des décisions humaines et des forçages naturels (Gaucherel et Baudry, 2004; Gaucherel et al., 2004a). Par comparaison, si la plateforme CORMAS (Bousquet et al., 1998) gère particulièrement bien les processus socio-économiques et décisionnels à travers son système multi-agents, elle se concentre essentiellement sur la communication entre agents pour modéliser les dynamiques spatio-temporelles des activités humaines (Tyssot et al., 2005) et non sur l'évolution spatio-temporelle des composantes du paysage. De même, si le modèle CLUE (Veldkamp et Fresco, 1996; Verburg et al., 2002) gère particulièrement bien les relations multi-scalaires (modélisation de décisions politico-économiques, évènements climatiques, etc.), il ne permet pas d'actions géométriques (telles qu'un arasement de haie, une réunion de parcelles, etc.) et semble par corollaire peu adapté à restituer des scénarios d'évolution de modes d'occupation des sols à une échelle fine, celle de la parcelle.

La généricité de la plateforme L1 lui permet d'être actuellement utilisée dans plusieurs domaines : la foresterie (Gaucherel et al., 2004b), l'écologie (flux de population) et l'écologie du paysage (Gaucherel et al., sous presse; Viaud, 2004). L'application dédiée à la modélisation de l'évolution d'un paysage agricole bocager utilise des données spatiales et temporelles. Les premières décrivent les composantes du paysage (parcellaire, bocage, occupation du sol, degré d'hydromorphie, limites spatiales et système de production des exploitations). Les secondes sont destinées à représenter les dynamiques d'évolution de ces composantes (évolution des exploitations, vitesse d'agrandissement du parcellaire, évolution des assolements par système de production, successions culturales, évolution de la densité bocagère, localisation et nombre d'arasements et de créations de haies, vitesse de fermeture et évolution de l'extension spatiale des zones humides de fonds de vallées). La simulation dynamique est issue de la réalisation, à un pas de temps annuel, de l'ensemble ou d'une partie des processus d'évolution des paysages agricoles bocagers (successions culturales, création/arasement de haies, reprise d'exploitation, etc.) et de leurs interactions spatiotemporel-

5. La cartographie des exploitations agricoles du Lestolet est soumise à une clause de confidentialité. 
les. Par exemple, la reprise d'une exploitation par une exploitation voisine, va engendrer l'année suivante une modification des successions culturales. Ces processus (reprise d'exploitation et successions culturales) interviennent à deux échelles différentes (parcellaire, exploitations agricoles). La localisation précise dans un espace géographique permet à chacune des parcelles de connaître l'exploitation englobante à laquelle elle appartient et son système de production, sa distance par rapport au siège d'exploitation, et ainsi, par corollaire, de déterminer le type de succession culturale va intervenir sur chacune d'entre elles. Le paysage résultant du premier pas de temps va être ensuite utilisé comme paysage initial (t) au pas de temps (année) suivant (fig. 3). Cette application peut être considérée, d'après la classification de T. Cheng (2005), comme un spatio-temporal object model, où l'approche objet ne tient pas dans la structuration informatique (orienté-objet) mais dans les relations spatiales entre les objets spatiaux (relation de voisinage - topologie -, relation multi-scalaire - englobé/englobant -).

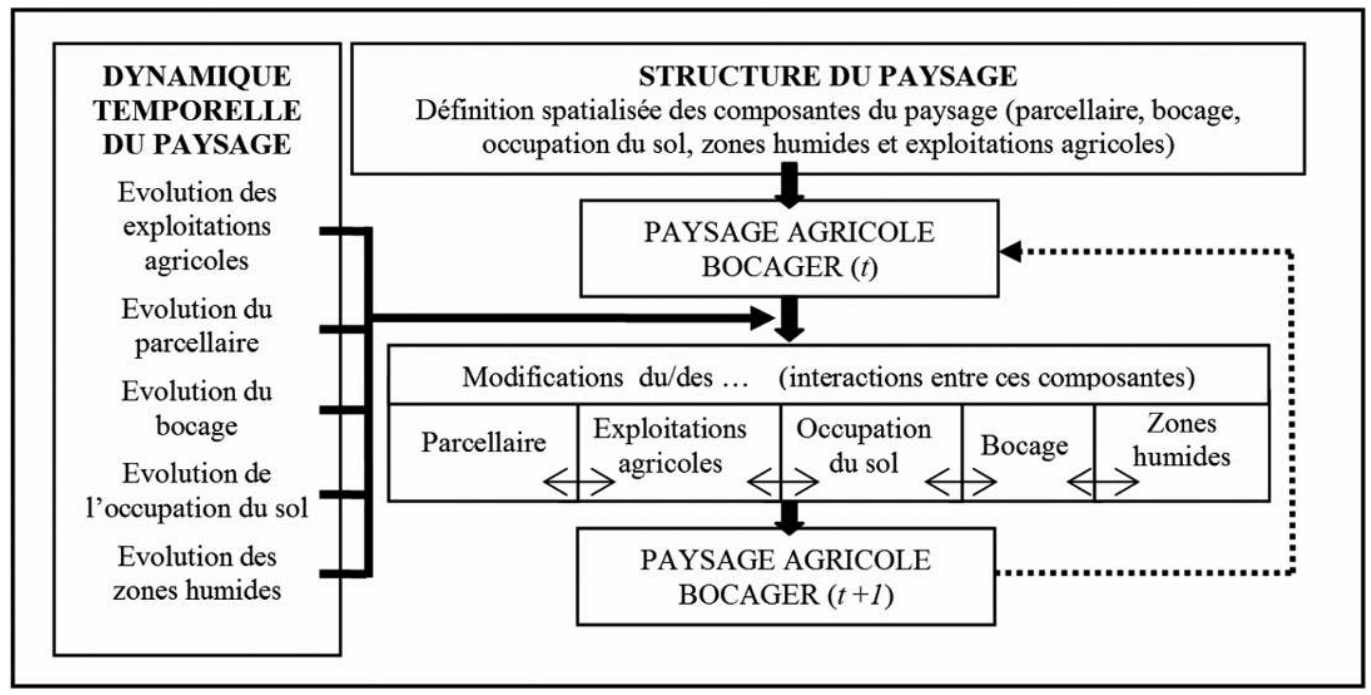

Figure 3 : Fonctionnement général de l'application dédiée à l'évolution d'un paysage agricole bocager de la plateforme $\mathrm{Ll}$

General functioning of the pacthy landscape modelling module of the L1 platform

Une étude récente (Houet et Gaucherel, sous presse) a permis d'évaluer la sensibilité et la robustesse du modèle en simulant l'évolution passée des structures paysagères et des modes d'occupation des sols sur le bassin versant du Lestolet sur une période de 18 ans (1981-1998). La comparaison spatiale et quantitative des changements simulés avec les changements réels observés a montré que L1 reproduit des dynamiques d'évolution et une organisation spatiale de la mosaïque paysagère de façon cohérente. La validation de la plateforme Ll a permis d'ouvrir le passage vers le domaine exploratoire par la simulation de scénarios prospectifs qui est exclusivement centrée sur l'occupation des sols dans cet article.

\section{Règles de définition des assolements}

On peut considérer que, pour chaque système de production (spécialisé «lait » intensif, spécialisé « lait» extensif, mixte « lait + porc », etc.), un exploitant va utiliser ou chercher à utiliser ces terres de façon relativement similaire chaque année. Cette utilisation vise à la fois à assurer le bien-être animal (pâtures), la production de fourrages et/ou de cultures nécessaires à l'alimentation des cheptels et enfin de produire des cultures destinées à la vente. La définition d'assolements 
moyens pour chacun des systèmes de production a été réalisée à partir de données de télédétection récentes $(1999,2002)$, de relevés de terrain $(2003,2005)$ et d'enquêtes auprès des agriculteurs (Geslot, 2005; Menasseri, 2004). Il s'agit de valeurs moyennes, peu représentatives de la diversité des situations (d'un site à un autre, d'une exploitation à l'autre, d'une année sur l'autre) provenant potentiellement de contraintes climatiques (sécheresse, etc.), agronomiques (successions culturales) et structurelles (lourds investissements, etc.).

Cette application a bénéficié de développements quant à la structuration des successions culturales dont le principe de fonctionnement est décrit par T. Houet et C. Gaucherel (sous presse). Dans la précédente version, un seul grand type de successions était appliqué aux différents systèmes de production. Cela suffisait à représenter de façon simplifiée l'assolement annuel des systèmes de production « bovin lait/viande » et « mixte à base bovine », mais était inadapté aux systèmes de production spécialisés ( porcin » et «bovin extensif»). Dans cette version, les successions sont différenciées en fonction des systèmes de production et de la spécificité des rotations pratiquées (fig. 4). Ces successions culturales ont été élaborées à partir de données de télédétection sur la période 1996-2002 sur trois sites en Bretagne dont le Lestolet (Hubert-Moy et al., 2003), à partir des travaux de S. Corgne (2005) et V. Viaud (2004) ainsi que d'un partenariat scientifique avec l'INRA-Sad Armorique. Pour chacun des systèmes de production, les successions culturales peuvent varier en fonction de la distance au siège d'exploitation, du précédent cultural, de la taille et du degré d'hydromorphie de la parcelle.

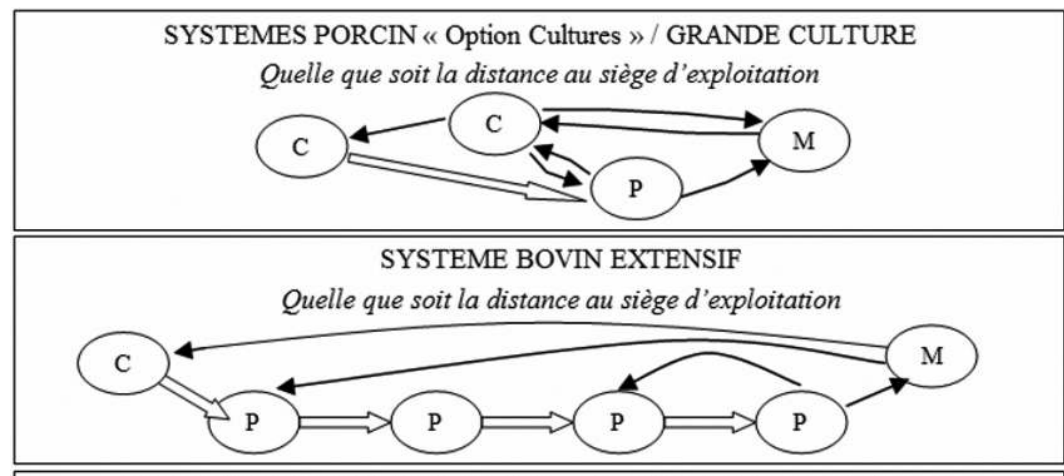

SYSTEMES BOVIN LAIT/VIANDE ou MIXTE / AUTRES SYSTEMES

Zone proche (Tiers de la SAU le plus proche du siège d'exploitation)

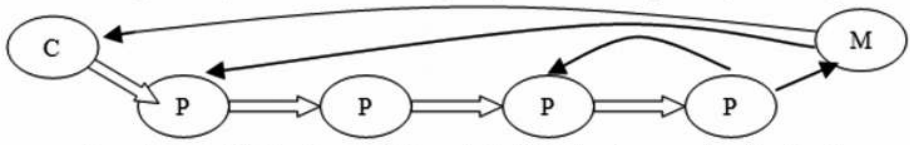

Zone intermédiaire (second tiers de la SAU le plus proche du siège)

Figure 4 : Définition des successions culturales par système de production

Crops successions for each system of production

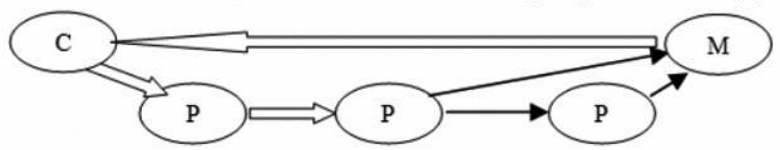

Zone éloignée (Tiers de la SAU le plus éloigné du siège)

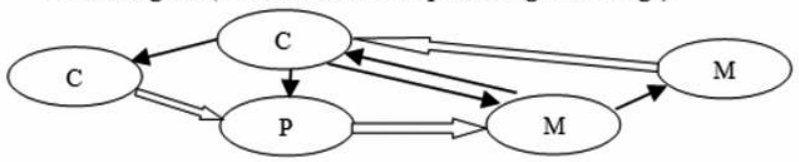

Succession obligatoire $\mathrm{P}$ : Prairie temporaire $\quad \mathrm{M}$ : Maīs 


\section{LES SCÉNARIOS PROSPECTIFS}

Trois simulations sont réalisées sur le Lestolet. Elles débutent en 1998, date à laquelle des données exhaustives sont disponibles, et projettent une image jusqu'en 2020 à un pas de temps annuel. Elles traduisent spatialement trois scénarios prospectifs qui ont été construits avec des experts et des acteurs locaux.

\section{Scénario A ou "le scénario de référence»}

Le premier scénario, appelé «scénario A », consiste à prolonger les tendances actuelles. Il se fonde sur les hypothèses peu vraisemblables que la réforme n'ait pas lieu (et donc que les assolements n'évoluent pas) et que les structures foncières des exploitations agricoles restent les mêmes jusqu’en 2020. Le scénario A constitue le scénario référent.

Scénario B ou "l'influence de la réforme de la PAC de 2006 »

Le second, dénommé «scénario B » illustre la mise en place de la PAC en 2006 sur le Lestolet. Les exploitations bovines présentes en 1998 sont relativement grandes et possèdent quasiment toutes des bâtiments mis aux normes. Ces caractéristiques soutiennent l'hypothèse d'une réforme favorable à l'implantation aux prairies modifiant fortement les assolements théoriques de l'ensemble des systèmes de production (tableau 1). Les céréales disparaissent au profit des prairies.

L'implantation d'herbe à la place du blé est une opération d'autant plus rentable que, tendanciellement, le prix du blé en Europe baisse pour répondre aux exigences du GATT et s'aligner sur les cours internationaux. Les économies proviennent des moindres coûts de traitement engendrés, de l'arrêt de location de matériels agricoles ou du recours à des ETA pour les semis et les récoltes. De plus, ils disposent déjà du matériel nécessaire à la gestion minimale des surfaces en herbe (fauche annuelle). Ils gagnent aussi beaucoup de temps libre, exigence qui devient de plus en plus forte dans la profession. Les structures foncières des exploitations n'évoluent pas durant la simulation.

L’hypothèse d'une modification généralisée des assolements en 2006 par l'ensemble des exploitants est simplificatrice. Toutefois la comparaison des scénarios A et B présente l'intérêt de mettre en évidence l'influence de la réforme de la PAC de 2006 suivant une hypothèse très contrastée. Ainsi, il est probable que la situation future se situe dans l'intervalle de ces deux situations.

\begin{tabular}{|c|c|c|c|c|c|c|}
\hline \multirow{2}{*}{ Systèmes de production } & \multicolumn{2}{|c|}{ Assolement avant 2006 } & \multicolumn{3}{c|}{ Assolement après 2006 } \\
\cline { 2 - 6 } & $P$ & $M$ & $C$-OP & $P$ & $M$ & $C$-OP \\
\hline Bovin lait et/ou viande & $65 \%$ & $20 \%$ & $15 \%$ & $80 \%$ & $20 \%$ & $0 \%$ \\
\hline Mixte bovin + volaille & $55 \%$ & $30 \%$ & $15 \%$ & $70 \%$ & $30 \%$ & $0 \%$ \\
\hline Mixte bovin + porc & $70 \%$ & $15 \%$ & $15 \%$ & $85 \%$ & $15 \%$ & $0 \%$ \\
\hline Porcin « option cultures » & $10 \%$ & $10 \%$ & $80 \%$ & $90 \%$ & $10 \%$ & $0 \%$ \\
\hline Volaille & $35 \%$ & $55 \%$ & $10 \%$ & $45 \%$ & $55 \%$ & $0 \%$ \\
\hline Bovin extensif / bio & $90 \%$ & $5 \%$ & $5 \%$ & $95 \%$ & $5 \%$ & $0 \%$ \\
\hline
\end{tabular}

$$
\mathrm{P} \text { : Prairies } \quad \mathrm{M} \text { : Maïs } \quad \mathrm{C}-\mathrm{OP} \text { : Céréales / Oléo-protéagineux }
$$

Tableau 1 : Évolution des assolements par système de production suite à la réforme de la PAC en 2006 (d'après Houet et Gaucherel, sous presse)

Evolution of landcover proportions for each system of production after the 2006 CAP reform (from Houet and Gaucherel, in press) 


\section{Scénario C ou "l'impact de l'agrandissement des exploitations »}

Le troisième scénario ne fait évoluer que les structures foncières des exploitations. Les assolements existants en 1998 ne changent pas tout au long de la simulation. Le nombre d'exploitations sur les trois communes concernées a fortement régressé entre 1979 et 2000 (-60\% en moyenne) engendrant un agrandissement croissant de leur taille moyenne (22 ha en 1979 contre 51 ha en 2000 [ONIC, 1979; ONIC, 2000]). D’après les données disponibles (âge des exploitants, SAU, etc.), il a été estimé que 17 exploitations sur les 31 existantes en 1998 cesseront leur activité d'ici 2020. Sur ces 17 cessations, 6 exploitations sont reprises par un jeune et les 11 autres par des exploitations voisines. Elles ont lieu entre 2001 et 2016: 1 reprise en 2001, 1 en 2002, 1 en 2004, 3 en 2007, 3 en 2008, 2 en 2009, 1 en 2010, 1 en 2012 et 3 en 2016. La taille moyenne des exploitations sera alors d'environ 90 ha mais avec de forts contrastes entre les " grandes » exploitations (100 à 130 ha) et les «petites » exploitations (environ 50 à 60 ha).

Les observations récentes montrent que l'agrandissement d'une exploitation se réalise essentiellement soit (1) par une reprise de la totalité d'une exploitation proche, soit (2) par une reprise de quelques parcelles suite au démembrement d'une exploitation. Pour des raisons informatiques, la modélisation de l'agrandissement des exploitations a été simplifiée et s'effectue par la reprise de la totalité d'une exploitation dès lors que son exploitant approche de l'âge de la retraite (estimé entre 55 et 60 ans). L'exploitation repreneuse est géographiquement proche et son exploitant relativement jeune (23-40 ans). Enfin, les reprises déterminées par les experts poursuivent la tendance observée d'une spécialisation vers la production « bovin lait/viande » conformément aux scénarios de l'Institut de l'élevage (2004).

Ces hypothèses sont simplificatrices : elles ne tiennent pas compte des situations économiques des exploitations (faute de données disponibles) et n'envisagent pas de reprise progressive des parcelles d'une exploitation qui est démembrée après cessation d'activité par plusieurs exploitations voisines. Elles présentent cependant l'intérêt d'évaluer l'effet d'un mouvement généralisé de reprise d'exploitations de proche en proche. L'influence de l'agrandissement de la structure foncière des exploitations est évaluée en comparant les scénario A et C.

\section{Résultats}

L'influence respective de la réforme de la PAC et de l'agrandissement foncier des exploitations sur l'évolution de l'occupation du sol d'ici 2020 a pu être quantifiée et spatialisée grâce aux simulations dynamiques et spatialement explicites réalisées avec la plateforme L1.

\section{L'IMPACT DE LA PAC 2006}

Conformément au scénario B, la réforme de la PAC 2006 se traduit par une augmentation de la part de prairies à l'échelle du bassin versant. Les changements générés semblent plus forts que ceux engendrés par la réforme de la PAC de 1992 (Houet et Gaucherel, sous presse). La simulation B permet d'évaluer quantitativement l'impact de la PAC à l'échelle du bassin versant : la proportion de prairies passe de 43,9\% de la surface totale du bassin versant avant 2006 à 52,3\% en moyenne entre 2007 et 2020, soit une hausse d'environ 116 ha (fig. 5b-planche III). La part moyenne de maïs avant 2006 était de $17,5 \%$ et atteint 13,8 \% après la réforme. La part de céréales restante après 2006 est $2,2 \%$, contre $7,7 \%$ avant la réforme.

La comparaison de la simulation A avec la simulation B (fig. 5a et 5b - planche III) témoigne de l'influence explicite de la PAC 2006. Dans le premier cas, les proportions d'occupation des sols présentent une faible variabilité interannuelle due aux rotations culturales. Dans le second cas, les répercussions de la réforme de la PAC 2006 sont sensibles et quasi-immédiates : les prairies se généralisent sur des espaces qui étaient en maïs ou en céréales avant la réforme. Les céréales ont quasiment totalement disparues. 
La distribution spatiale des cultures (fig. $6 a$ et $6 \mathrm{~b}$ - planche IV) est similaire entre les scénarios A et B : elles se localisent aux mêmes endroits sur le bassin versant en 2008 et 2018, de façon majoritairement dispersée, avec toutefois la présence de quelques petits îlots de parcelles cultivées.

Suite à la PAC de 2006, la place croissante des prairies (temporaires et permanentes) dans l'assolement tend à ralentir légèrement la fermeture des zones humides de fonds de vallées. Quelques parcelles de prairies permanentes ${ }^{6}$ ne sont plus abandonnées et n'évoluent plus en friches dans le scénario B comparativement au scénario A (99 ha de prairies permanentes en 1998; 85 ha en 2020 pour le scénario A contre 82 ha dans le scénario B).

\section{L'IMPACT DE L'AGRANDISSEMENT DES EXPLOITATIONS AGRICOLES}

La spécialisation croissante dans la production «bovin lait/viande » de ce secteur issue de l'agrandissement des exploitations agricoles tend à diminuer légèrement la part de prairies de 43,9\% en 1998 à environ 42 \% en 2020 (fig. 5c - planche III). Dans le même temps, la part en maïs est restée pratiquement constante (autour de $18 \%$ ) et celle des céréales a légèrement augmenté (de 7,7 \% à 8,9\%). Ainsi, les reprises foncières contribuent à augmenter de près de 20 ha entre 1998 et 2020 la surface en céréales à l'échelle du bassin versant.

Cette restructuration foncière des exploitations a principalement des répercussions sur la localisation des cultures (maïs et céréales). La comparaison des scénarios A et $\mathrm{C}$ (fig. 6a et 6c-planche IV) montre une tendance au regroupement des parcelles cultivées en îlots au sein d'une exploitation. Ceci est accentué par la juxtaposition de parcelles de cultures de diverses exploitations qui sont repoussées dans les auréoles les plus lointaines du modèle concentrique qui se met en place autour des bâtiments d'élevage. Par conséquent, les haies situées autour de ces parcelles représentent une gêne potentielle importante pour les engins agricoles et une surface non négligeable pour augmenter les rendements, les rendant particulièrement exposées au risque d'arasement.

Enfin, l'agrandissement foncier tend à accélérer la fermeture des zones humides de fonds de vallées (120,5 ha de friches et de bois en 1998; 134,7 ha en 2020 pour le scénario A contre 143,4 ha dans le scénario C). Cela se justifie par deux facteurs : les exploitants sont de moins en moins nombreux augmentant ainsi la charge de travail nécessaire à l'entretien des prairies humides; les parcelles humides d'une exploitation venant d'être reprise se situent désormais potentiellement plus loin de leur nouveau siège, augmentant ainsi le risque d'abandon.

\section{LA LOCALISATION DE ZONES STRATÉGIQUES}

\section{POUR UNE GESTION DURABLE ET EFFICACE DE L'EAU}

La culture du maïs constitue une plante à risque vis-à-vis de la qualité de l'eau puisqu'elle n'est pas sensible à une sur-fertilisation et qu'elle peut parfois engendrer l'absence de couverture hivernale des sols favorable aux transferts de flux polluants de surface (phosphore, pesticides) et de sub-surface (nitrates). Les cartographies annuelles des modes d'occupation des sols produites par la plateforme L1, permettent de réaliser des cartes de fréquence de retour en maïs pour les trois scénarios prospectifs (fig. 7 - planche V).

La localisation des zones présentant une forte fréquence de retour constitue un outil d'aide à la gestion durable et efficace de l'eau, d'une part car il tient compte de la longue durée, d'autre part car ces zones sont localisées finement et enfin parce qu'il intègre plusieurs hypothèses aux conséquences contrastées. Par ailleurs, la localisation de celles situées à proximité des cours d'eau peut permettre aux gestionnaires de l'eau ou acteurs agricoles de mieux sensibiliser à des pratiques raisonnées les exploitants qui les utilisent.

6. Leur faible nombre provient du fait que les exploitants susceptibles d'utiliser ces parcelles humides n'ont pas tous été identifiés. Elles n'ont donc pas toutes été prises en compte dans la simulation et le calcul des types d'occupation du sol dans les zones humides. 
La localisation des haies situées à proximité des parcelles occupées par du maïs au moins une année sur deux, apporte là encore des informations précieuses pour une meilleure gestion de l'eau. Ces haies sont susceptibles d'être arasées alors même qu'elles peuvent avoir un rôle tampon essentiel vis-à-vis de la qualité de l'eau.

\section{Bilan et discussion}

La modélisation prospective de l'occupation des sols effectuée sur le Lestolet a permis de montrer que la réforme de la PAC de 2006 et l'agrandissement des exploitations en zone agricole intensive spécialisée dans l'élevage bovin ont des incidences différenciées sur l'évolution des modes d'occupation des sols à l'échelle d'un bassin versant à l'horizon 2020. Les simulations dynamiques, fondées sur trois scénarios prospectifs, ont montré de façon quantifiée et spatialisée l'ampleur, le rythme et la répartition spatiale des évolutions de l'occupation du sol pour chacun des scénarios : la réforme de la PAC de 2006 peut tendre vers une augmentation sensible des surfaces en herbe; à l'inverse, l'agrandissement des exploitations tendrait à légèrement accroître la part de cultures.

La mise en évidence des impacts respectifs de la réforme de la PAC de 2006 et de l'agrandissement des exploitations agricoles permet désormais d'envisager la construction d'un scénario prospectif spatialisé les intégrant simultanément. Un tel scénario interroge sur l'influence cumulée et antagoniste de ces deux facteurs sur l'évolution de la proportion des prairies à l'échelle du bassin versant, ainsi que sur le devenir et la vitesse de fermeture des zones humides de fonds de vallées.

La simulation dynamique et spatialement explicite de scénarios prospectifs, à l'aide de la plateforme Ll met aussi en évidence des espaces stratégiques à l'échelle d'un bassin versant pour la gestion de la ressource en eau. Elle permet de localiser (1) des zones où la pression sur la structure bocagère est forte, (2) des zones à risque vis-à-vis des transferts de flux polluants où des couverts hivernaux doivent être préconisés en raison de fortes fréquences de retour en maïs et enfin, (3) des parcelles à risque fort localement et dans le long terme en raison de leur proximité au réseau hydrographique et de leur fortes fréquences de retour et où les conseils en matière de pratiques raisonnées prennent toute leur importance. D’autres espaces présentant des enjeux importants, tels que les parcelles humides où le risque d'abandon et de fermeture peut réduire les potentialités dénitrifiantes des zones humides de fonds de vallées, pourraient être mis en évidence en comparant la situation initiale avec la situation finale.

Toutefois, même si cette plateforme est particulièrement adaptée pour la modélisation paysagère à l'échelle locale, elle est encore à l'état de prototype qui peut être optimisée, notamment sur l'inertie de la chaîne de calcul. Il faut entre 1 h 15 et 3 heures pour réaliser chacun des scénarios. Si elle permet de modéliser de façon simplifier les décisions humaines et les forçages naturels à l'aide de règles déterministes, stochastiques et/ou téléonomiques laissant peu de place à l'incertitude, il apparaît que le couplage avec un Système Multi-Agent peut contribuer à améliorer la modélisation.

Enfin, si certaines hypothèses des scénarios prospectifs peuvent apparaître un peu trop simplifiées, la traduction de leurs incidences dans le temps sur un territoire en offre une vision qui n'en est pas moins plausible. L'exploration de l'incertitude liée à l'évolution à long terme à travers l'expérimentation d'hypothèses complémentaires et/ou contrastées (peu probables mais envisageables) constitue une démarche essentielle pour aider à une gestion durable de l'eau. Les simulations ne cherchent pas à prédire l'avenir avec exactitude mais permettent de localiser et de quantifier les marges de manœuvres existantes. La méthode des scénarios prospectifs spatialisés à l'échelle locale constitue un outil puissant d'aide à la décision pour les gestionnaires de l'eau afin de mieux cibler et orienter les conseils auprès des agriculteurs et les aménagements à réaliser. 


\section{Bibliographie}

Agreste Bretagne, 1998. - 1970-1997 La mutation de l'agriculture bretonne, Rennes, Direction Régionale de l'Agriculture et de la Forêt, $n^{\circ} 28$, octobre 1998, 25 p.

—, 2001. - «Recensement agricole 2000. Premiers résultats », Ed. Direction Régionale de l'Agriculture et de la Forêt, mai 2001, 4 p.

Baudry (J.), Burel (F.), Aviron (S.), Martin (M.), Ouin (A.), Pain (G.), Thenail (C.), 2003. - "Temporal variability of connectivity in agricultural landscape: do farming activities helps?”, Landscape Ecology, vol. 18, n 3, p. 303-314.

Bousquet (F.), Bakam (I.), Proton (H.), Le Page (R.), 1998. - «Cormas: Common-pool Resources and Multi-Agent Systems », Lecture notes in Artificial Intelligence, vol. 1416, p. 826-837

Cheng (T.), 2005. - Multi-scale spatio-temporal data modeling, analysing and reasoning, Habilitation à Diriger des Recherches, Université de Strasbourg/Sun Yat-Sun University (China), 191 p.

Corgne (S.), 2005 - « Hiérarchisation des facteurs de changements de l'occupation hivernale des sols Application au bassin versant du Yar (Bretagne) », Norois, vol. 193, p. 17-29.

Gaucherel (C.), Baudry (J.), Auclair (D.), Houlller (F.), "Landscape modelling : the state of the art", Landscape Ecology, (soumis).

Gaucherel (C.), Baudry (J.), 2004. - Application de L1 à un paysage agronomique réaliste, Présentation de la réunion bilan, 25 novembre 2004, [http://www.inra.fr/efpa/internet/animations/PlateformePaysage/L 1BilanB.pdf].

Gaucherel (C.), Baudry (J.), Auclair (D.), 2004a. - La modélisation du paysage et la plateforme L1, Présentation de la réunion bilan, 25 novembre 2004, [http://www.inra.fr/efpa/internet/animations/PlateformePaysage/L1-BilanA-base.pdf].

Gaucherel (C.), Fleury (D.), Auclair (A.), Baudry (J.), Dreyfus (P.), 2004b. - "Forest modeling based on landscape ecology concepts. Landscape ecology of trees and forests", Proceedings of the twelfth anmual IALE (UK) conference, p. 309-312.

Gaucherel (C.), Giboire (N.), Viaud (V.), Houet (T.), Baudry (J.), Burel (F.), 2006. - « A domain specific language for patchy landscape modelling : the Brittany agricultural mosaic as a case study », Ecological Modelling, vol. 194, n 1-3, p. 233-243.

GesLot (S.), 2005, L'incidence de la réforme de la PAC 2006 sur les systèmes de production agricole bretons et sa répercussion sur l'occupation des sols, Rapport de MASTER 2 Aménagement du Territoire - Option Gestion de l'Environnement, Université de Rennes 2 Haute-Bretagne, 111 p.

Houet (T.), Hubert-Moy (L.), 2004. - Étude diagnostique de l'évolution de l'occupation des sols sur trois sousbassins versants du Blavet (1952-2002), Rapport Final, COSTEL, Rapport interne, 95 p.

Houet (T.), Gaucherel (C.), « Simulation dynamique et spatialement explicite d'un paysage agricole bocager : Validation sur un petit bassin versant breton sur la période 1981-1998 », Revue Internationale de Géomatique, (sous presse).

Hubert-Moy (L.), Houet (T.), Corgne (S.), 2003. - Étude diagnostique de l'évolution de l'occupation des sols sur trois sous-bassins versants du Blavet (1952-2002) : Rapport intermédiaire, COSTEL, Rapport interne, 96 p.

INSEE, 1982 - Recensement Général de la Population, Données statistiques, CD-Rom.

—, 1999 - Recensement Général de la Population, Données statistiques, CD-Rom.

INSTITUT DE L'ÉLEVAGE, 2004, Réforme de la PAC et production laitière : scénarios d'évolution à l'horizon 20102012, Le Dossier économie de l'élevage, Paris, Institut de l'élevage, 70 p.

Menasseri (S.), 2004. - Enquêtes sur les conduites des cultures dans les exploitations agricoles du bassin versant de Naizin (Morbihan) en 2004, Rapport de synthèse + Enquêtes réalisées par les étudiants du DAA Ingénierie des Agrosystèmes et du DESS Productions végétales - Option Sciences Agronomiques, Agrocampus Rennes, 19 p. (+ Annexes)

Mermet (L.), Poux (X.), 2002 - Pour une recherche prospective en environnement : repères théoriques et méthodologiques, Natures, Sciences, Sociétés, vol 10, n³, p. 6-14.

ONIC, 1979. - Recensement Général Agricole, Données statistiques, CD-Rom.

—, 2000. - Recensement Général Agricole, Données statistiques, CD-Rom. 
Poux (X.), 2003. - « Les méthodes de scénarios » dans Mermet (L.) (dir.), Prospectives pour l'environnement: Quelles recherches? Quelles ressources? Quelles méthodes?, Paris, Documentation française, coll. «Réponses environnement », p. 33-50.

Poux (X.), Mermet (L.), Bouni (C.), Narcy (J.-B.), Dubien (I.), 2001. - Méthodologie de prospective des zones humides à l'échelle micro-régionale - problématique de mise en ceuvre et d'agrégation des résultats, Rapport scientifique au PNRZH, $111 \mathrm{p}+$ annexes.

Thenail (C.), 1997. - « Le rôle des exploitations agricoles dans l'évolution des paysages : un facteur essentiel des dynamiques écologiques », Ecologia Mediterranea, vol. 23, p. 71-90.

Thenail (C.), BAudry (J.), 2001. - « Modélisation des systèmes techniques agricoles contribuant aux dynamiques des structures paysagères : de la parcelle à l'exploitation agricole et au paysage », Etude des changements d'utilisation et d'occupation du sol: échelles et modèles, Séminaire de l'UMR 6554, Rennes, L. Hubert-Moy (éd.), p. 16-24.

Tyssot (C.), Le Tixerant (M.), Rouan (M.), CuQ (F.), 2005. - « Modélisation spatio-temporelle des activités humaines à fort impact environnemental », Cybergéo, n³13, p. 1-15.

Veldkamp (A.), Fresco (L.), 1996. - « CLUE: a conceptual model to study the Conversion of Land Use and its Effects », Ecological Modelling, vol. 85, p. 253-270.

Verburg (P.), Veldiamp (A.), Soepboer (W.), Limpiada (R.), Espaldon (V.), Mastura (S.), 2002. - « Modeling the spatial dynamics of regional land use: the CLUE-S model », Environmental Management, vol. 30, n³, p. 391-405.

VIAUD (V.), 2004. - Organisation spatiale des paysages bocagers et flux d'eau et de nutriments, Thèse de doctorat, École Nationale Supérieure Agronomique de Rennes, 281 p.

Cet article a été reçu le 16 décembre 2005 et définitivement accepté le 27 mars 2006.

\section{REMERCIEMENTS}

L'auteur tient à remercier la chambre d'agriculture des Côtes-d'Armor, le Syndicat Mixte de Kerné Uhel et l'Institution Interdépartementale du SAGE Blavet pour les données mises à disposition et pour leur participation aux réunions visant la réalisation des scénarios prospectifs. Que J. Baudry (INRA-Sad Armorique), Xavier Poux (AsCA/RGTE-ENGREF) et Bernard Clément (ECOBIO) soient également remerciés à ce titre ainsi que pour leur appui méthodologique. Enfin, j’adresse ma reconnaissance aux relecteurs pour leurs conseils avisés et pertinents. 
Planche I (Thomas HOUET - Modélisation prospective de l'occupation du sol...)
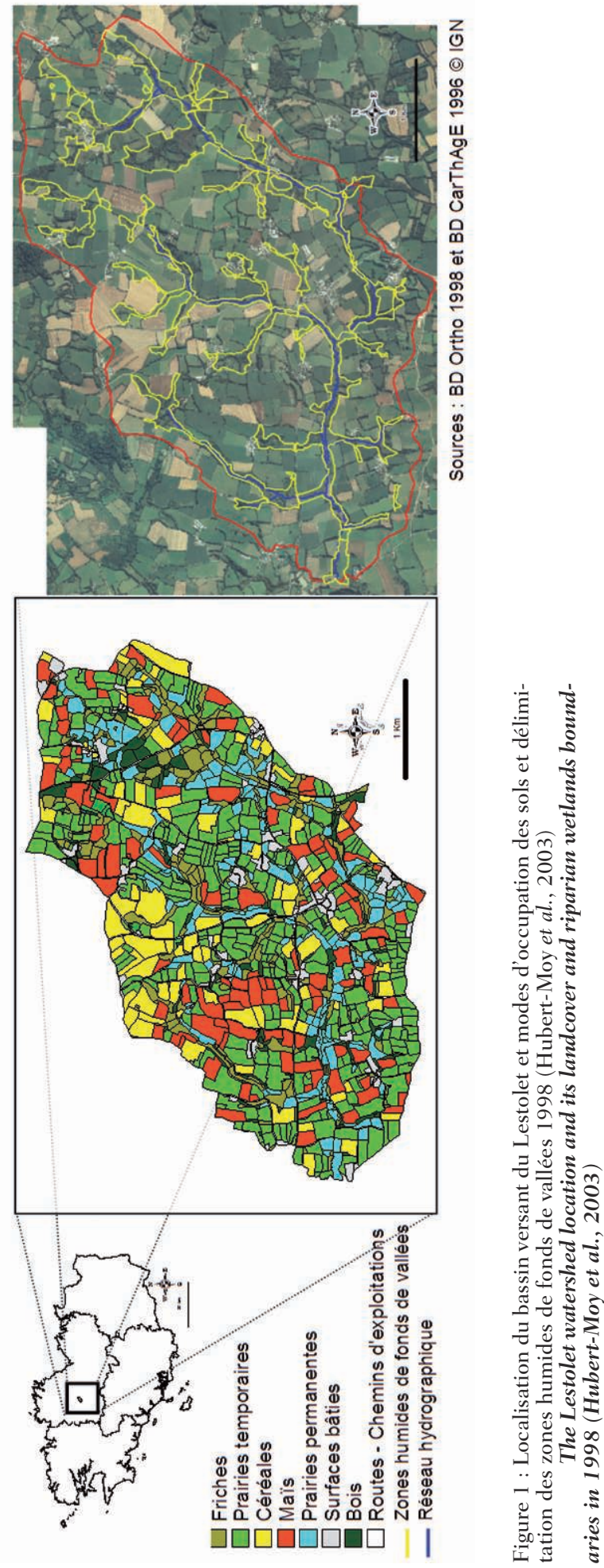
Planche II (Thomas HOUET - Modélisation prospective de l'occupation du sol...)

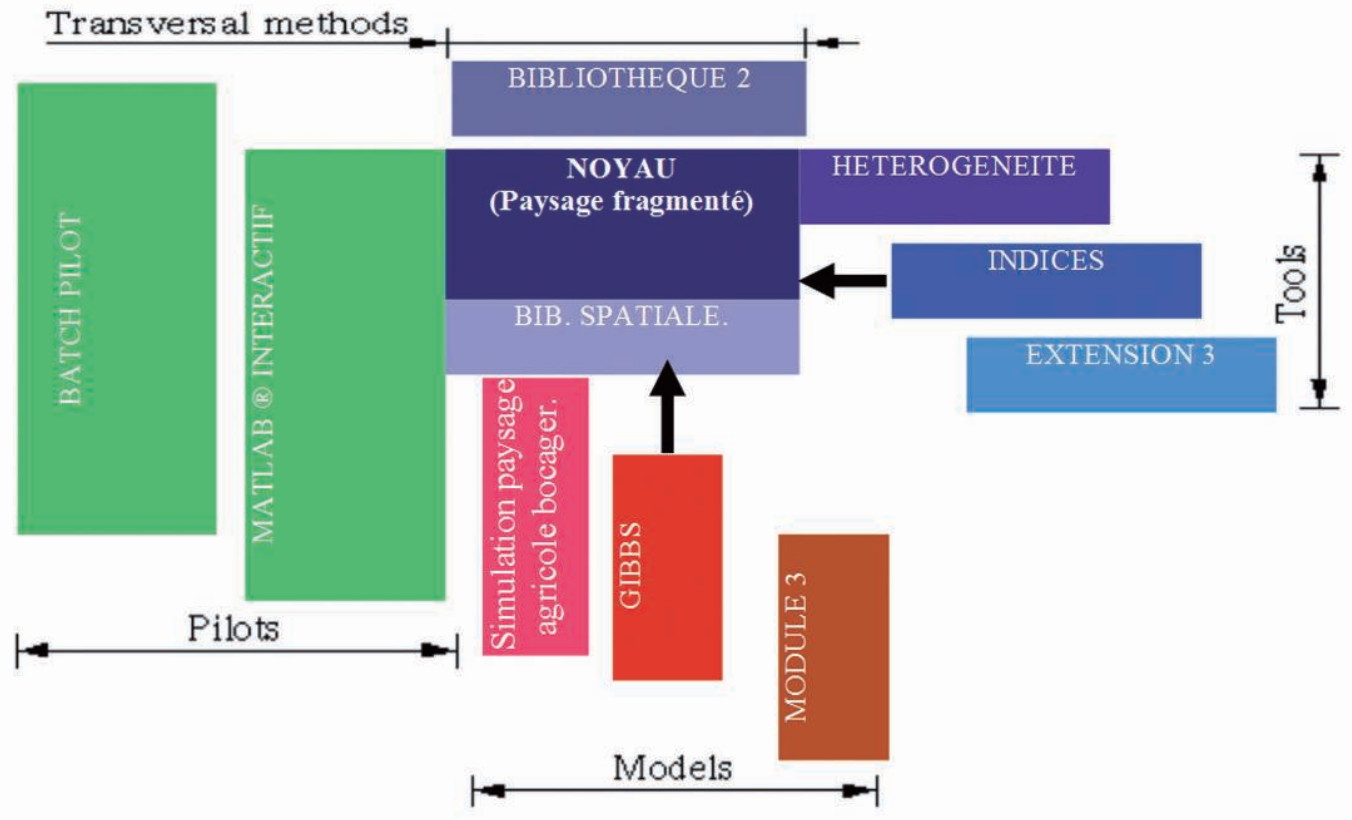

Figure 2 : Architecture de la plateforme Ll (dans Gaucherel et al., 2004a, adapté) L1 platform architecture (dans Gaucherel et al., 2004a, adapted) 
Planche III (Thomas HOUET - Modélisation prospective de l'occupation du sol...)

(a)

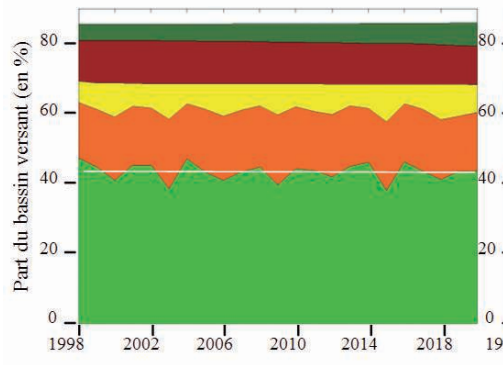

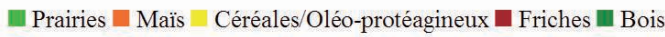

(c)

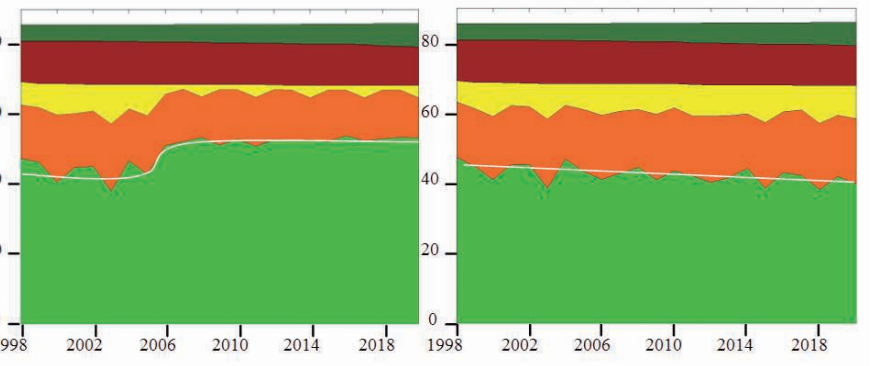

Figure 5 : Évolution de la proportion des types d'occupation du sol à l'échelle du bassin versant dans le cas du (a) scénario A, (b) scénario B et (c) scénario C (c) scenario $C$

Evolution of landcover proportions at the watershed scale for the (a) scenario $A,(b)$ scenario $B$ and 
Planche IV (Thomas HOUET - Modélisation prospective de l'occupation du sol...)

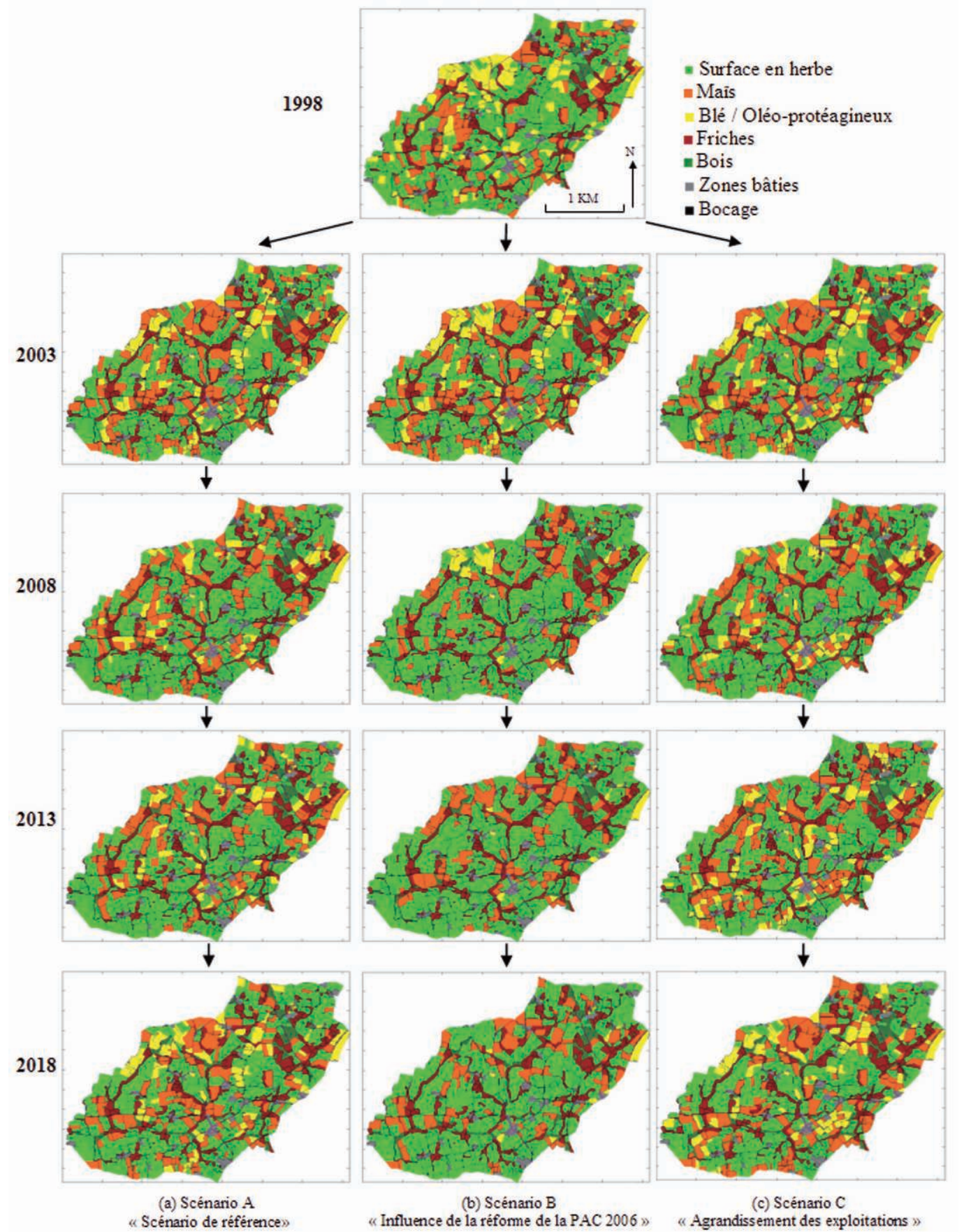

Figure 6 : Évolution des modes d'usage des terres en 1998, 2008 et 2018 à l'échelle du bassin versant dans le cas du (a) scénario A, (b) scénario B et (c) scénario C

Evolution of land use and landcover in 1998, 2008 et 2018 at the watershed scale in the (a) scenario $A$, (b) scenario B et (c) scenario $C$ 
Planche $\boldsymbol{V}$ (Thomas HOUET - Modélisation prospective de l'occupation du sol...)

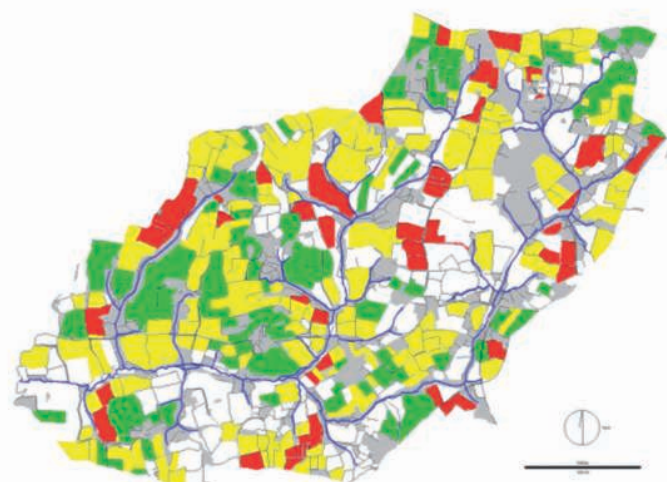

(a)

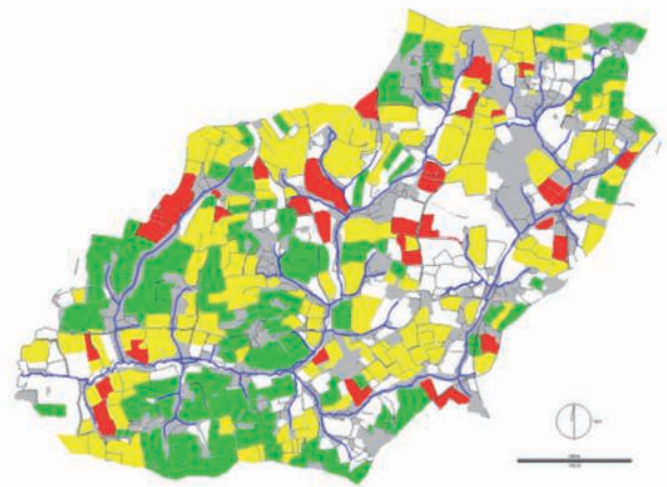

(c)

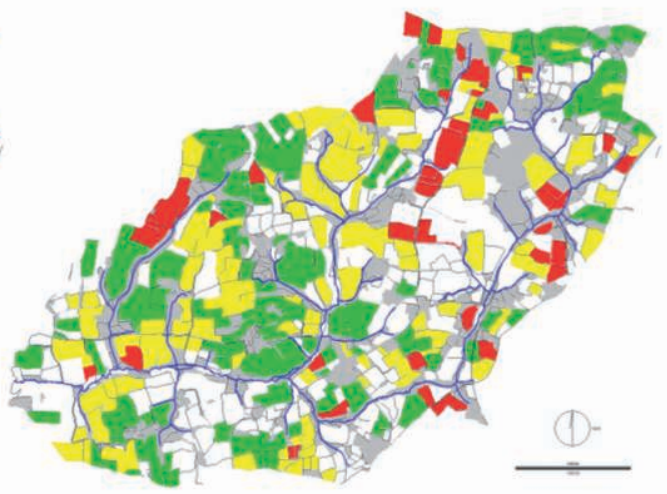

(b)

\section{LEGENDE}

Fréquence de retour en maiss

Aucune implantation de maïs

Présence d'un maiis moins d' 1 année sur 3

Présence d'un maïs moins de 2 années sur 3

Présence d'un maîs plus de 2 années sur 3

Réseau hydrographique

Bocage

Figure 7 : Localisation des espaces stratégiques de gestion de l'eau dans le cas du (a) scénario A, (b) scénario B et (c) scénario $\mathrm{C}$ scenario $C$

Localization of strategic water management areas for the (a) scenario A, (b) scenario B and (c) 